\title{
The Inverse Analysis of Concrete Fracture Property Based on Evolution- ary Algorithm
}

\author{
Zhigang Zhao ${ }^{1}$ and Zhifang Zhao, ${ }^{2 *}$ \\ ${ }^{I}$ School of New Media, Zhejiang University of Media and Communications, Hangzhou Zhejiang, China; ${ }^{2}$ College of \\ Civil Engineering and Architecture, Zhejiang University of Technology, Hangzhou Zhejiang, China
}

\begin{abstract}
As the softening constitutive relation of concrete basic fracture properties (softening curve), limited by the current level of experimental techniques, the test methods of softening curve such as direct tension tests and so on are difficult, high cost and different outcomes, so the study is difficult to move forward. In recent years, the inverse analysis study which uses numerical methods to determine the softening curve based on simple fracture test has emerged. The author combines concrete direct tensile test and the whole process of inverse analysis by numerical method, which is engaged in many years, and discusses the new ideas for solving the softening curves by using of computer technology to select the optimized method. The softening curve obtained by inverse analysis with the thinking of programming and the curve obtained by experiment are in line within the range of allowable error.
\end{abstract}

Keywords: Closed program, evolutionary algorithm, inverse analysis, softening curve.

\section{INTRODUCTION}

Methods for obtaining the softening curve of concrete include direct tensile method, $\mathrm{J}$ integral method and the inverse analysis method based on the simplified fracture test. For the fracture test with $\mathrm{J}$ integral method to determine the softening curve, the tensile strength should take the partial differential between the energy consumption for fracture formation and preset (CTOD), whereas, it is very difficult to obtain the accurate CTOD and the energy consumption quantity for fracture formation. It is very hard to realize it in regular laboratories since this experiment is full of technical difficulties. From the curve for direct stress-deformation $(\sigma-\delta)$ obtained from the direct tensile test, we can get the $\sigma-w$ curve (softening curve) between the necessary for us to make the geometric and mechanical centering for both the test specimen and the tester, to prevent eccentric tension of the test specimen. But when the test specimen is cracked, it is possible to incur eccentric tensile due to the asymmetry of early-stage fracture. The test result for the stress is only the average of the whole load-carrying surface, the tested deformation value $(w)$ is the only deformation or fracture width of the surface of the test specimen, which makes the softening curve inaccurate. Therefore, we can predict that it will be more difficult to obtain an ideal result through the direct tensile test.

\section{THE INVERSE ANALYSIS OF CONCRETE SOF- TENING CURVE}

Key point for determine the softening curve with the inverse analysis method is, to get the softening curve with the inverse analysis by test data obtained from the simple concrete fracture test-wedge splitting tensile test (WS test) and the cohesive fracture mode (CCM). See Fig. (1) for WS test:
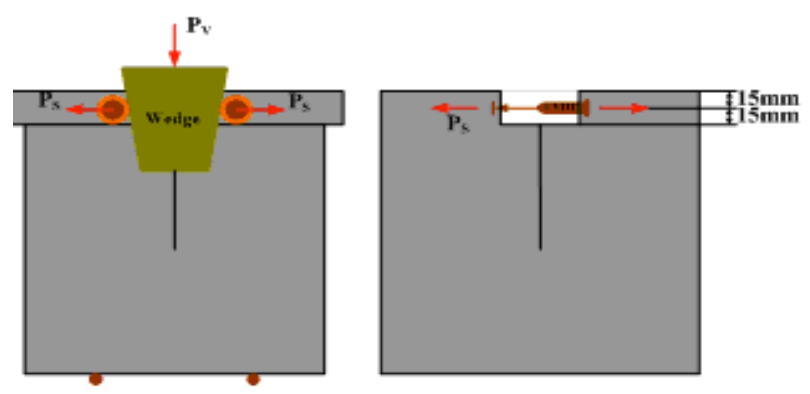

Fig. (1). Wedge splitting tensile test (WS) of concrete.

In this thesis, test data obtained by the load-crack mouth opening displacement (P-CMOD) will be adopted based on the evolutionary algorithms to conduct the inverse analysis on the softening curve. Establish a model with finite elements for the WS test specimen, select appropriate formation and parameters for the softening curve, adopt the error function to work out the softening curve[1]. Its train of thoughts is shown in Fig. (2) [2], among which, the error function e is the deviation between the P-CMOD actually tested and the P-CMOD value obtained by simulation.

Division of finite-element grid, selection of error function and the adopted optimization algorithm are the three primary factors for conducting this inverse analysis.

\subsection{Numerical Finite-element Model}

Parameters for the softening curve can be obtained through testing or through numerical simulation [3]. Working out the softening parameters with inverse analysis is a kind of numerical simulation. Through the inverse analysis calculation based on the CCM mode and the actual curve 
tested through the WS, we can determine the multi-stage linear softening curve.

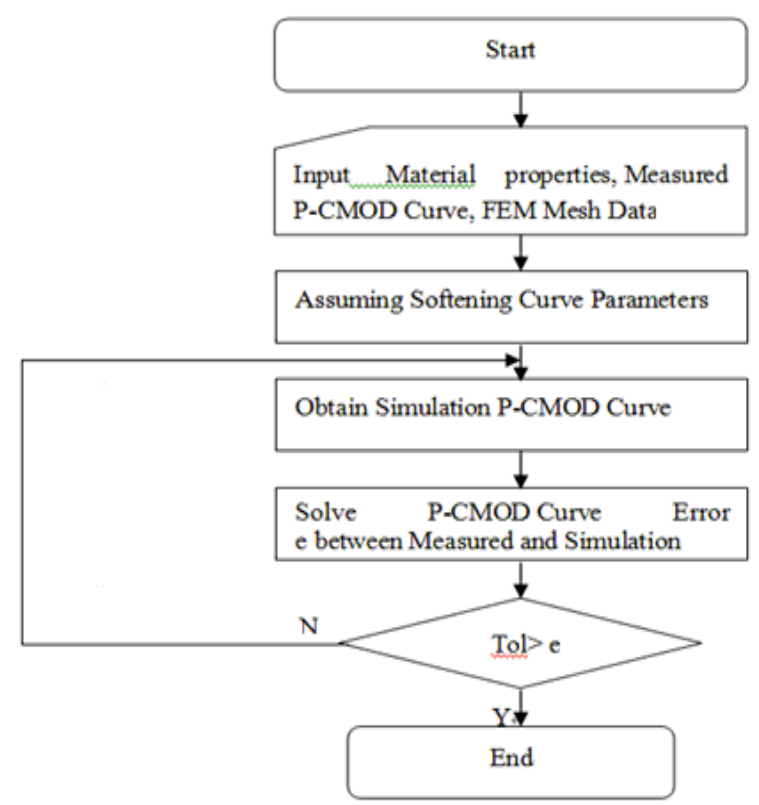

Fig. (2). Inverse analysis flow chart.

Suppose the other area except for the fracture process zone (FPZ) is elastic in the fracture expansion process of WS test. Within the finite-element mode (FEM) process, set joint constraints along the direction of fracture expansion (see Fig. 3 ), if we release the joint constraints, the fracture will continue to go ahead. Based on the CCM mode, exert cohesive force towards the FPZ at the fracture tip already existing, in order to transform the non-linear to such a boundary with cohesive force. This kind of simplification can help solve the non-linear problems with superposition principle without iteration over and over again in the finite-element calculation process.

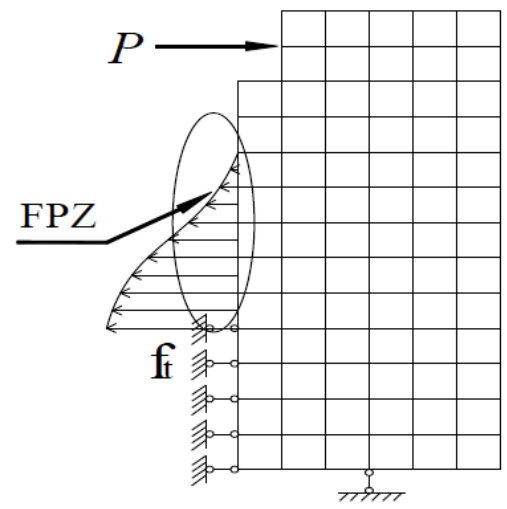

Fig. (3). Fracture extension simulation of WS test specimen.

Within the FEM simulation, when the stress of the joint constraints at the fracture extension direction reaches the fracture strength $f_{t}$, release the joint constraints to make the fracture go forward. With the extension of the fracture, the joint constraints will be released one by one along the extension direction.

\subsection{Determination of the Actually Tested Representative P-CMODa Curve for Inverse Analysis}

Treat the P-CMODa curve preliminarily treated with smoothing matching through quadratic spline approximation method [4] to obtain representative curves for around 200 points, which will be used as input data for the inverse analysis, introduce the smoothing parameter $\lambda$, adjust the matching result manually to obtain the most appropriate representative curve of $\mathrm{P}-\mathrm{CMODa}$.

Selection of $\lambda$ work should be conducted carefully and subject to the actually tested P-CMODa curve [5]. Smoothing of the curve is not asked to be as perfect as possible, since we need to control the error within a limit. This process has been integrated into the inverse analysis programme and does not require for independent treatment.

\subsection{Grid Division Format}

To promote the speed for fracture simulation is critical to use the finite element for simulation. For numerical simulation, to simplify a problem into a plane stress problem, we only need to make analyses on half of the integrate structure since it is symmetrical. For WS test, grid division format in Fig. (4) can be adopted. In this way, DOF of this structure can be reduced visibly and efficiency can be improved. But the geometric dimensions of the test specimen must be input in advance, besides, we need to propose the stiffness equation of the materials for test specimen as a kind of linear elasticity.

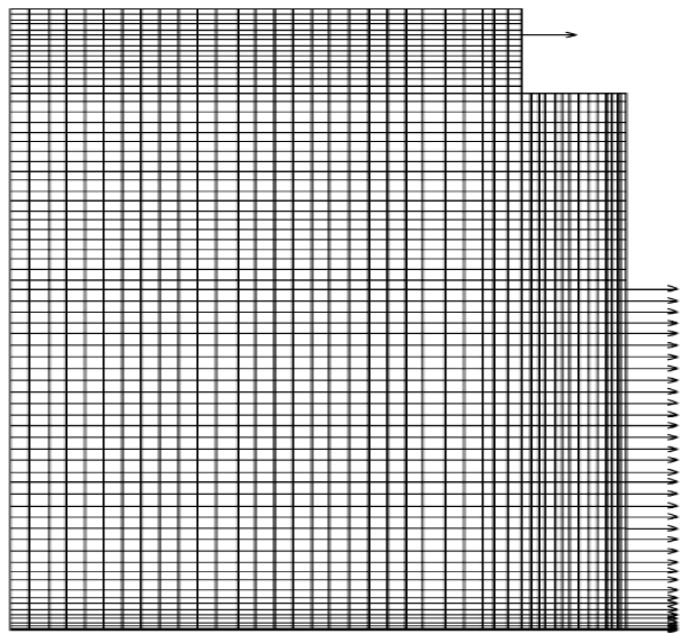

Fig. (4). Grid division format for finite element of WS test specimen.

\subsection{Error Function e}

Selection of error function is a premise for optimization and can influence the final result of optimization. In order to calculate the deviation generated in the inverse analysis process, we need to make the discrete P-CMODa curve actually tested into an approximately smooth curve. Since dispersion degree for each group of actual data is different, the two-stage spline approximation treatment method for parameter $\lambda$ should be adopted. There are 3 forms of error function for you to select: 
The average relative error [6]

$$
e \mathrm{l}=\sum_{i=1}^{n} \frac{\left|\arctan \left(m_{i} / k\right)\right|}{90^{\circ}} \cdot\left|\frac{f_{i}-y_{i}}{m_{i} \cdot \bar{x}}\right|+\left\{1-\frac{\mid \arctan \left(m_{i} / k\right)}{90^{\circ}}\right\} \cdot\left|\frac{f_{i}-y_{i}}{\bar{y}}\right|
$$

where, $\left(x_{i}, y_{i}\right)$ is the point on the P-CMODb curve for numerical simulation, whereas, $\left(x_{i}, f_{i}\right)$ is the point on $\mathrm{P}$ CMODa curve actually tested;

$$
y=A x^{2}+B x+C
$$

where, $\mathrm{A}, \mathrm{B}$ and $\mathrm{C}$ is obtained through points

$$
\begin{aligned}
& P_{i-1}\left(x_{i-1,} y_{i-1}\right), P_{i}\left(x_{i}, y_{i}\right) \text { and } P_{i+1}\left(x_{i+1}, y_{i+1}\right) ; \\
& m_{i}=\left.\frac{d y}{d x}\right|_{i}, \forall i \in[2, n-1], m_{1}=\frac{y_{2}-y_{1}}{x_{2}-x_{1}}, i=1, \\
& m_{n}=\frac{y_{n}-y_{n-1}}{x_{n}-x_{n-1}}, i=n \text { and } \frac{\left|\arctan \left(m_{i} / k\right)\right|}{90^{\circ}}
\end{aligned}
$$

are weighting functions; when $\mathrm{k}$ is relatively small $(\mathrm{k} \approx 1)$, $\mathrm{el}$ is the relative error at the $\mathrm{X}$-axis (displacement) direction; when $\mathrm{k}$ is very big $(\mathrm{k}>\mathrm{E})$ relative to tangent modulus $\mathrm{E}$, el is the relative error at the $\mathrm{Y}$-axis (load) direction;

\section{Maximum Relative Error e2}

$$
e 2=\max \left\{\frac{\left|\arctan \left(m_{i} / k\right)\right|}{90^{\circ}} \cdot\left|\frac{f_{i}-y_{i}}{m_{i} \cdot x}\right|+\left\{1-\frac{\left|\arctan \left(m_{i} / k\right)\right|}{90^{\circ}}\right\} \cdot\left|\frac{f_{i}-y_{i}}{\bar{y}}\right|\right\}
$$

\section{Relative Error e3 for Breaking Energy[7]}

$$
\begin{gathered}
e 3=\frac{1}{2}\left(\frac{G_{F \exp }-G_{F n u m}}{G_{F \exp }}+\frac{G_{F \exp }-G_{F n u m}}{G_{F n u m}}\right) \\
G_{F \exp }, G_{F n u m} \text { are breaking energy [7] values }
\end{gathered}
$$

obtained respectively by actual test and numerical simulation, this is a situation for

$G_{\text {Fnum }} \rightarrow 0, G_{\text {Fnum }} \rightarrow \infty$.

Generally the error function of e $1+\mathrm{e} 3$ is recommended. In case the actually tested P-CMODa curve is not integrate, e3 will not be accepted as the error function.

\subsection{Optimization Algorithm}

Optimization of parameters to the softening function with the actually tested P-CMODa curve, is a kind of non-trivial problem within a mathematic problem, which has no analytical solution. Otherwise, we can view the optimization problem as working out the minimum value of an error function. Given the complicity of FCM mode, it is very difficult to deduce the gradual explicit formulation of an error function. It's necessary to introduce human intervention and adjustment in the optimization process, but the corresponding result will be influenced by human subject factors. The author has made a lot of efforts in searching for more appropriate parameter variation procedures, the best solution was selected finally by stochastic optimization. During the circulating curve fitting, multi-local minimum values still exist within the error function, in other words, multi-solutions exist for the softening function. Based on observation to the direct tensile test, and considering the similarity of related materials, a solution which has physical meaning and is plausible and real can be worked out.

Key points for selecting the optimization algorithm are to avoid the local minimum value and to obtain a physically meaningful solution, and to promote the efficiency. Among them, the stochastic optimization method is the best of all for this study. Simulated annealing is a common way of stochastic optimization, whereas, its convergence rate is relatively too low, besides, local minimum can be easily introduced. In this thesis, the evolutionary algorithm will be adopted as the optimization method for inverse analysis.

The evolutionary algorithm [8] is a kind of artificial intelligent algorithm based on the biological evolutionary theory and has developed innovatively, it is a kind of popular and highly-efficient stochastic searching and optimization method. It is a kind of dynamic integration between the genetic algorithm and evolutionary strategy. It is developed from the inheritance, mutation, natural selection and hybridization of evolutionary biology. It searches from the bunch of the problem solutions, which is the obviously difference between it and the traditional optimization algorithm. The latter gets the optimal resolution from single initial value iteration. It searches from the bunch, covers more spheres and is beneficial for the integrate prioritizing. At the same time, it can deal with many other individuals within the same group and make evaluations to the multi-solutions within the searching space, thus, reducing the risk of locally optimal solution. Besides, it is easy for the algorithm itself to realize parallelization. Expanding the evolutionary algorithm with such local search principle can improve convergence rate, and reduce risk of local minimum regarding obtaining the fitness function, which is good for optimization.

Combining with the direct tensile test, and based on the simple rupture test and the inverse analysis based on Levenberg-Marquardt [9] algorithm for virtual fracture model, the thesis carries out fitting of non-linear curve to determine the softening relation between the concrete of bulk massive and natural gradation and the wet sieve concrete. Based on the LM algorithm, problems occurred in the matching process of P-CMOD curve (softening curve can be determined by fitting) have been analyzed and integrated into the genetic algorithm to conduct optimization selection and improve curve matching and prioritizing effects.

More specifically, the evolutionary algorithms mainly used in the process of data comparison. By comparing over and over again with $r$, the sum of square of deviation between the polynomial value obtained through the curve equation by matching result of the least square method and the function value $\mathrm{yj}$, to get the minimum rj, then, make the reciprocal of the square sum of the residual error as the fitness, which is divided into the following steps: encoding of the curve expression frequency, foundation of the initial group, calculation of the fitness value, generation of new chromosome through genetic recombination via selection, intersect and variation, evaluation to the new chromosome, modify the generation system (calculation of new fitness value and replacement of parent chromosome per corresponding principles) to give a new group. Determined if it is in line with 
Table 1. 6 Softening parameters obtained for the WS test specimen group by inverse analysis calculation.

\begin{tabular}{|c|c|c|c|c|c|c|}
\hline Test piece & $f_{t}$ & $f_{1}$ & $f_{2}$ & $w_{1}$ & $w_{2}$ \\
\hline \hline WS-1 & 2.546 & 2.052 & 0.472 & 0.0083 & 0.1130 \\
\hline WS-2 & 2.203 & 1.601 & 0.412 & 0.0025 & 0.1173 & 0.4907 \\
\hline WS-3 & 2.531 & 1.704 & 0.434 & 0.0020 & 0.1225 & 0.4688 \\
\hline
\end{tabular}

suspension conditions, if yes, then shut down the computer and pick out the global optimum and corresponding input value, if not, continue with a new round of circulation.

\subsection{Average of Softening Curve}

6 parameters can be calculated out for three linear softening curve by the above inverse analysis. As Table $\mathbf{1}$.

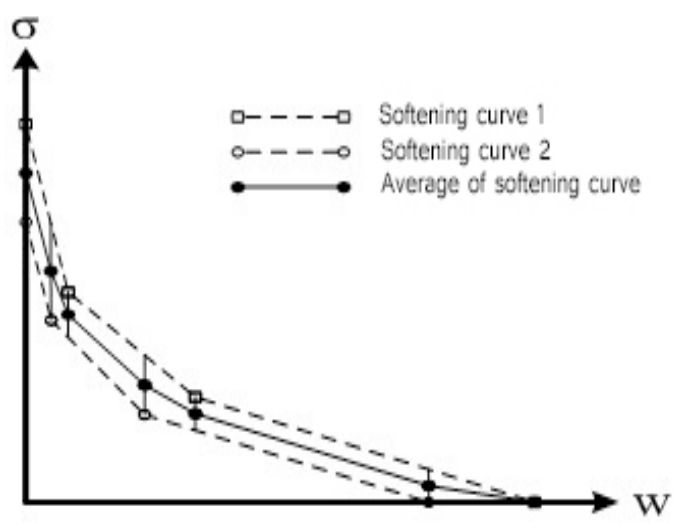

Fig. (5). Average of softening curve.

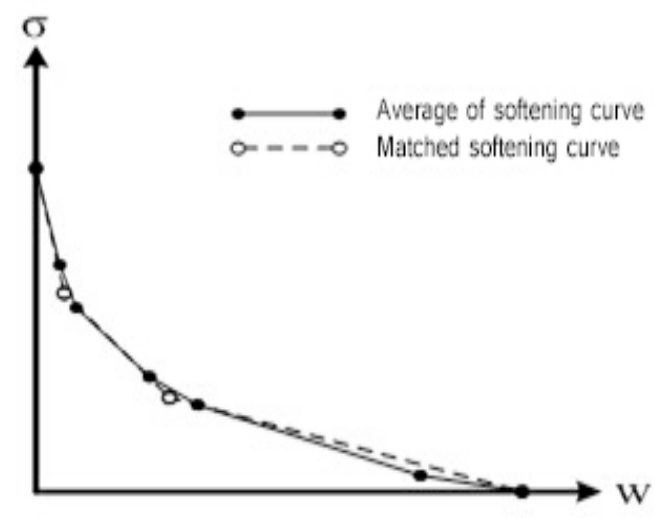

Fig. (6). Matching diagram for softening curve average.

Fig. (5) shows the averaging method for the softening curve. Get the average of all stress values $\sigma$ corresponding to each inflection point of each softening curves of all test specimen within the same group, get the maximum value of all softening curves as $w_{c}$. In this thesis, matching work for the softening curve after averaging will be completed by the 1 stOpt software, as shown in Fig. (6). This optimization process is targeted to make both values as consistent as possible on the premise that the under curve area for the polyline is identical.

\subsection{Softening Curve Parameters Obtained through In- verse Analysis}

See Fig. (7) and Table 2 for the 6 parameters of threestage linear softening curve of WS test specimen obtained through inverse analysis and corresponding rupture energy Gf.

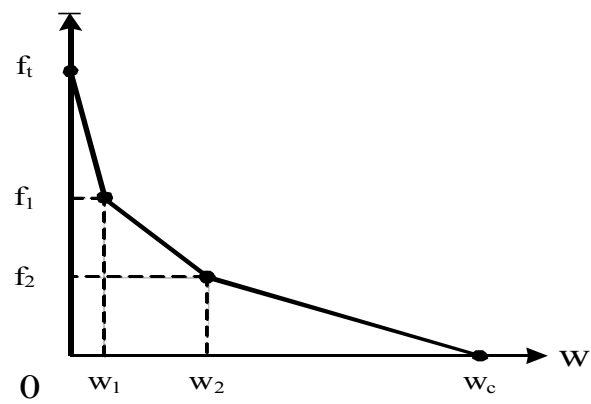

Fig. (7). Three-stage linear softening curve diagram.

\section{SIMULATION AND RESULT}

\subsection{Algorithm Description}

The evolutionary algorithms mainly used in the process of data comparison [10]. The ordinary procedure is encoding of the curve expression frequency, foundation of the initial group, calculation of the fitness value, generation of new chromosome through genetic recombination via selection, intersect and variation, evaluation to the new chromosome, modify the generation system (calculation of new fitness value and replacement of parent chromosome per corresponding principles) to give a new group. Determined if it is in line with suspension conditions, if yes, then shut down the computer and pick out the global optimum and corresponding input value, if not, continue with a new round of circulation.

In this article the program idea is divided into the following steps.

1. Receive each data of $X$ value and $Y$ value and deposited in two arrays.

2. Calculate the number of data and establish matrix of $X$ value and $\mathrm{Y}$ value.

3. Select the value of $m$ and use the Polyval function to derive curve expression.

4. Write $\mathrm{M}$ file of Matlab, $\mathrm{r} 2$ aj(fitness) and $\mathrm{p}$ (proportional ratio of fitness value).

5. Change the m, multiple calls to $M$ file, compared to aj (fitness). 
Table 2. 6 Parameters and fracture energy for three-stage linear softening curve of WS test specimen.

\begin{tabular}{|c|c|c|c|c|c|c|c|}
\hline Specimen & $f_{t}$ & $f_{1}$ & $f_{2}$ & $w_{1}$ & $w_{2}$ & $w_{c}$ & $\mathbf{G}_{\mathbf{f}}$ \\
\hline \hline WS2 & 2.337 & 1.576 & 0.400 & 0.0175 & 0.1619 & 0.7796 \\
\hline WS & 2.733 & 2.032 & 0.159 & 0.0042 & 0.1506 & 0.6700 \\
\hline 19 & 3.074 & 2.062 & 0.220 & 0.0051 & 0.4366 & 0.9269 \\
\hline WS & 2.990 & 1.541 & 0.235 & 0.0214 & 0.1813 & 0.6722 \\
\hline WS & 3.191 & 1.440 & 0.246 & 0.0260 & 0.2986 & 247.9 \\
\hline WS & & & & & 4.2358 \\
\hline
\end{tabular}

Change the value of $m$ is the use of a circular function, set the range of $\mathrm{m}$, calculate fitness of aj corresponding to different $\mathrm{m}$, then compare to keep the $\mathrm{m}$ of larger fitness [11]. Narrow the range of $m$ appropriately if it is found that the fitness decreases, then give the next cycle to contrast until meeting the necessary error conditions.

\subsection{Simulation and Analysis}

In this thesis, the inverse analysis application by matlab will be adopted. The Visual Basic is adopted to design a simple user interface.

Firstly, set data of P-CMODa curve for WS test specimen supplied by the test, then, input corresponding elasticity modulus and Poisson's ratio. When the 6 parameter calculation mode is selected, 6 three-stage linear softening parameters should be input. See Fig. (8).

In Fig. (9), curve that is consisted of pink points is the actually tested P-CMODa curve, whereas, curve that is consisted of red points is the P-CMODb curve obtained by inverse analysis calculation. Three primary softening parameters: $G_{F}, f_{t}, w_{c}$ for comparison in this thesis will be listed in corresponding textbox for related parameters. The two curve fits well.

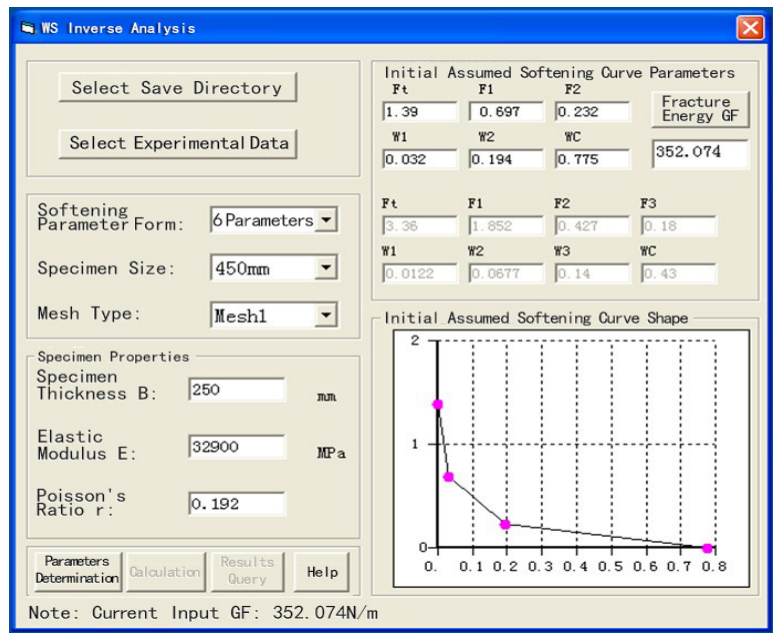

Fig. (8). Initial Inputs for WS Test Specimen.

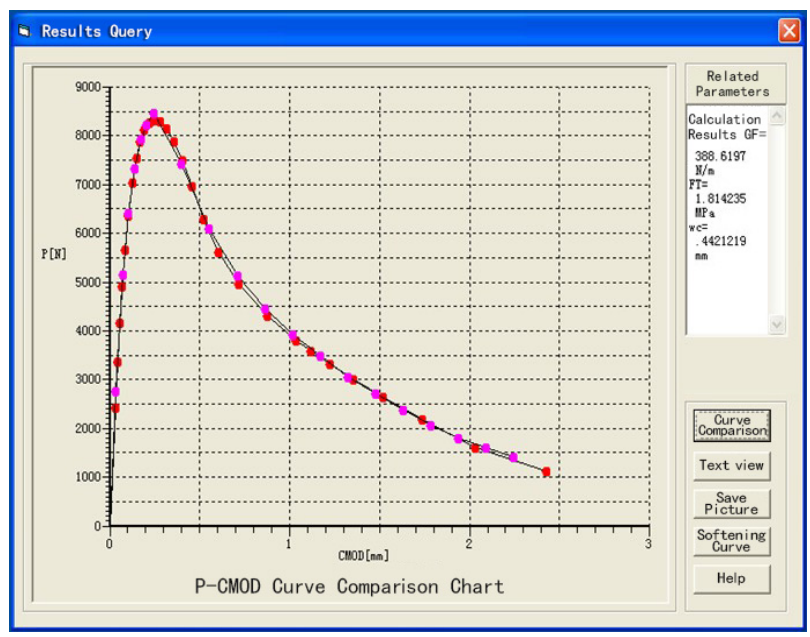

Fig. (9). Comparison between the two P-CMOD curve.

The softening curve determined through inverse analysis is shown in Fig. (10). By obtaining the comparison curves for various parameters, comparison and calculation for inverse analysis can be done more effectively.

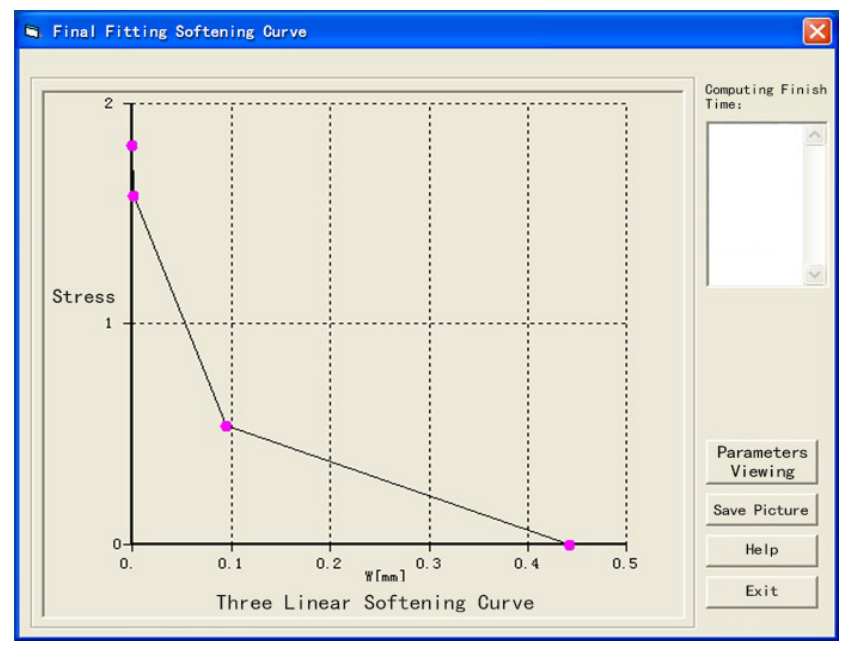

Fig. (10). Softening curve obtained by inverse analysis. 


\section{CONCLUSION}

In this thesis, determination of concrete softening constitutive relation by numerical simulation based on a series of simple tests has been studied. WS test is mainly adopted to get the actual P-CMODa curve. Based on it, the softening curve is also supposed, the P-CMODb curve is obtained by numerical simulation.

The key point is to introduce the local searching mechanism into the evolutionary algorithm, by comparing over and over again with $r$, the sum of square of deviation between the polynomial value obtained through the curve equation by matching result of the least square method and the function value $y j$, to get the minimum rj, then, make the reciprocal of the square sum of the residual error as the fitness, determine the number of the next generation by fitness until the number of offspring is less than the parent number, then get the final result.Fitting P-CMODa curve obtained from fracture test and $\mathrm{P}-\mathrm{CMODb}$ curve obtained from numerical simulation by optimized evolutionary algorithm, so that a most plausible softening curve can be obtained.

\section{CONFLICT OF INTEREST}

The authors confirm that this article content has no conflict of interest.

\section{ACKNOWLEDGEMENTS}

This work is supported by the National Natural Science Foundation of China (Project No.51479178), Zhejiang Provincial Natural Science Foundation of China (Grant No.Y14E090021), and the Colleges and Universities Visiting
Scholar Teacher Professional Development Program (FX2014069) of Zhejiang Province.

\section{REFERENCES}

[1] Zhifang Zhao, Seung Hee Kwon, Surendra P. Shah. Effect of specimen size on fracture energy and softening curve of concrete: Part I. Experiments and fracture energy [J]. Cement and Concrete Research, 2008, 38: 1049-1060.

[2] Yang LOU, Junli LI, Linpeng JIN, Gang LI, A Co Evolutionary Algorithm Based on Elitism and Gravitational Evolution Strategies, Journal of Computational Information Systems [J] 8: 7 (2012) 2741-2750.

[3] Zhao Fengzhi, Quadratic approximation method in numerical optimization [M]. Beijing: Science press, 2004.

[4] Liu Mandan, Research progress in cultural gene algorithms [J], Control theory and application, 2007, 26(11): pp. 1-5.

[5] Bazant, Z.P.,Concrete fracture models: testing and practice [J], Engineering Fracture Mechanics. 2002, 69: 165-205.

[6] V. Slowik, B. Villmann, N. Bretschneider, T. Villmann, Computational aspects of inverse analyses for determining softening curves of concrete, Computational Methods in Applied Mechanics and Engineering (2006), pp. 7223-7236.

[7] DUAN Kai, HU Xiao-zhi, FOLKER H. Wittmann size effect on specific fracture energy of concrete [J]. Engineering Fracture Mechanics, 2007 (74) : pp.87-96.

[8] Villmann, T. Evolutionary algorithms with subpopulations using a neural network like migration scheme and its application to real world problems [J], Integr. Comput. -Aided Engrg, 2002, 9: 25-35.

[9] K.M. Brown, Derivative-free analogues of the LevenbergMarquardt and Gauss algorithms for non-linear least square approximations [J], IBM, Philadelphia Scientific Center Technical Report, 1990: pp. 320-2994.

[10] M. Hauschild, M. Pelikan, K. Sastry, and Goldberg, D. E.(2012). Using previous models to bias structural learning in the hierarchical BOA. Evolutionary Computation, 20(1): pp. 135-160, 2012.

[11] Jiaqi Liu, Jixin Yang, Chunyan Yang, Evolutionary algorithm in least square method for curve fitting, Journal o f Computer Applications [J], 2010, 30(12): 40-42.

\footnotetext{
Received: May 26, 2015

Revised: July 14, 2015

Accepted: August 10, 2015

(C) Zhao and Zhao; Licensee Bentham Open.

This is an open access article licensed under the terms of the (https://creativecommons.org/licenses/by/4.0/legalcode), which permits unrestricted, noncommercial use, distribution and reproduction in any medium, provided the work is properly cited.
} 